\title{
Molecular scale analysis of dry sliding copper asperities
}

\author{
Bhavin N. Vadgama • Robert L. Jackson • \\ Daniel K. Harris
}

Received: 8 April 2014/ Accepted: 4 July 2014/Published online: 24 July 2014

(C) The Author(s) 2014. This article is published with open access at Springerlink.com

\begin{abstract}
A fundamental characterization of friction requires an accurate understanding of how the surfaces in contact interact at the nano or atomic scales. In this work, molecular dynamics simulations are used to study friction and deformation in the dry sliding interaction of two hemispherical asperities. The material simulated is copper and the atomic interactions are defined by the embedded atom method potential. The effect of interference, $\delta$, relative sliding velocity, $v$, asperity size, $R$, lattice orientation, $\theta$, and temperature control, on the friction characteristics are investigated. Extensive plastic deformation and material transfer between the asperities were observed. The sliding process was dominated by adhesion and resulted in high effective friction coefficient values. The friction force and the effective friction coefficient increased with the interference and asperity size but showed no significant change with an increase in the sliding velocity or with temperature control. The friction characteristics varied strongly with the lattice orientation and an average effective friction coefficient was calculated that compared quantitatively with existing measurements.
\end{abstract}

Keywords Adhesion - Friction mechanisms .

Nanotribology $\cdot$ Copper $\cdot$ Contact mechanics

\section{Background}

Understanding the physics of friction is of fundamental importance for a wide range of applications and more so for

B. N. Vadgama · R. L. Jackson $(\bowtie) \cdot$ D. K. Harris

Department of Mechanical Engineering, Auburn University, Auburn, AL 36849, USA

e-mail: jacksr7@auburn.edu small-scale applications. With the rapid development of surface examining technologies like AFM, FFM and others as well as development of MEMS and NEMS devices, a better understanding of the atomistic mechanisms of sliding friction is essential. When surfaces in contact slide across each other only a small number of micro or nano sized peaks or asperities truly come into contact. The manner in which these contacting asperities interact has a significant influence on the frictional characteristic of the sliding surfaces. The phenomena occurring at the nano-scale are therefore complex and difficult to predict since the tribological properties of sliding contacts are greatly affected by the adhesion and contact deformation. Furthermore, the adhesion is directly proportional to the number of atomic or molecular bonds that are broken and formed at the interface of contacting surfaces during sliding (Landman et al. 2004). Below a certain scale the dependence of the friction and dissipation of frictional heat on factors such as inter-atomic forces, surface topography and composition of materials increases (Achanta et al. 2009). Since the asperities or peaks on rough surfaces occur at multiple scales and may have contact areas with values scaling over many orders of magnitudes, their properties can vary significantly due to scale dependent mechanisms (Jackson 2006). Therefore, the behavior of asperities at the smaller scales may benefit from techniques such as molecular dynamics (MD) to characterize them. Blau (1991) has shown that steady-state friction is highly scale dependent and therefore the friction mechanisms should be modeled considering the entire tribosystem rather than a discrete asperity system. Such an exercise to model the scale effects would be extremely productive if carried out using MD, provided the computational resources allow.

Computer simulations, specifically using MD, have become quite popular among researchers with much work 
being done in micro and nano tribology. In one of the early works, Harrison et al. (1992) investigated the atomic-scale friction of diamond surfaces using MD. Landman et al. (1996) studied the formation and properties of interfacial junctions in the normal contact between a hemispherical asperity and a flat surface. They also study the shearing of lubricated junctions in asperity-asperity sliding geometry. In a later work (Landman et al. 2004), they reviewed the Amontons' Law with the help of MD and suggested that for adhering surfaces an additional contribution, which is proportional to the real molecular contact area, is present in the basic equation of friction. Song and Srolovitz (2007) performed MD simulations of single asperity contact and deformation on a flat surface over single- and multi-cycle loading and unloading. In an extensive study, Sorensen et al. (1996) conducted MD simulations on atomic-scale friction of sliding copper surfaces. The dependence of the normal load, contact area, sliding velocity, temperature, and lattice mismatch was investigated with an emphasis on observing slip-stick for different tip-surface and surfacesurface contacts. They observed atomic scale stick slip for matching as well as non-matching surfaces; however, it decreased with an increase in the contact size. Zhang and Tanaka (1997) performed MD simulations of a diamond tip sliding over a flat copper surface. They found that four distinct deformation regimes existed characterized by nowear, adhering, ploughing, and cutting of the surface. These were governed by indentation depth, sliding speed, asperity geometry, and surface conditions. Investigation of the simple case of a single asperity sliding over a flat surface using MD has also been carried out by several other research groups (Cho et al. 2005; Jeng et al. 2007; Yang and Komvopoulos 2005; Zhu et al. 2011; Zhang et al. 2001; Ivashchenko and Turchi 2006). However, an important aspect of the frictional sliding process between rough surfaces is the interaction between two separate asperities rather than an asperity and a flat surface. The current work studies two separate hemispherical asperities, which has surprisingly only been studied a few times using molecular dynamics.

It would be useful to study asperity-asperity interaction to gain useful insights on the deformation mechanisms and frictional characteristics in addition to the work done on the asperity-surface case as mentioned above. In the most similar work, Zhong et al. (2003) performed MD simulations of two asperities sliding into each other to study such asperity-asperity interaction. In their work, they modeled a sliding "hard" upper asperity deforming a fixed lower aluminum asperity, which is still very different from the two deformable copper asperities considered in the current work. They studied the effects of a wide range of conditions including sliding velocities, temperatures, and crystal orientations on the wear process. At the micro-scale, several continuum based semi-analytical as well as finite element based models have also been developed for friction between sliding asperities and cover both elastic as well as plastic deformations (Jackson et al. 2007; Faulkner and Arnell 2000; Boucly et al. 2007). Therefore, in addition, it would be interesting and insightful to see if these models can be compared to MD simulations at the nanoscale. There have also been recent works that blend molecular dynamics and the finite element method, but the current work uses only the MD method (Eid et al. 2011).

The origin of friction is an extremely complex phenomenon and is an open and growing research field. The existing theories developed for the bulk are not always consistent with material behavior at the molecular scale. The relationship between experimentally measured values of friction to the material properties is still not clear which makes the prediction of friction challenging. The objective of this work is to numerically study the atomistic mechanism of friction and contact deformation using molecular dynamics simulations on the dry sliding contact of nanoscale asperities. The material selected for this work is copper, which has been increasingly used in nano-engineering where metallic properties are of importance. The higher electrical and thermal conductivity along with low friction makes copper suitable for use in applications such as MEMS switches (Barriga et al. 2007) or as an additive to enhance the "wear mending" property of a lubricant (Liu et al. 2004). Besides its application in MEMS, copper is also the nano-material of choice to be incorporated in the friction material used in automotive brake pads for its ductility and high thermal conductivity (Osterle et al. 2010). Copper nanoparticles have been used as an additive to enhance the tribological properties of lubricants (Tarasov et al. 2002; Choi et al. 2009). The effects of interference $\delta$, relative sliding velocity $v$, asperity size $R$, lattice orientation $\theta$, and temperature control, on the friction characteristics are investigated quantitatively and qualitatively. All the simulations were carried out using the MD code LAMMPS (Plimpton 1995).

\section{Methodology}

The geometric 3D model used here to represent asperityasperity sliding contact is shown in Fig. 1. The model consists of upper and lower sections each having a hemispherical asperity and a rigid base, composed of copper atoms in a FCC lattice structure. The $x, y$, and $z$ axes are oriented in the [100], [010] and [001] lattice directions, respectively. Periodic boundary conditions are imposed along the $x$ - and $z$-axis so that the atoms can exit one side 
Fig. 1 Asperity-asperity sliding contact simulation model

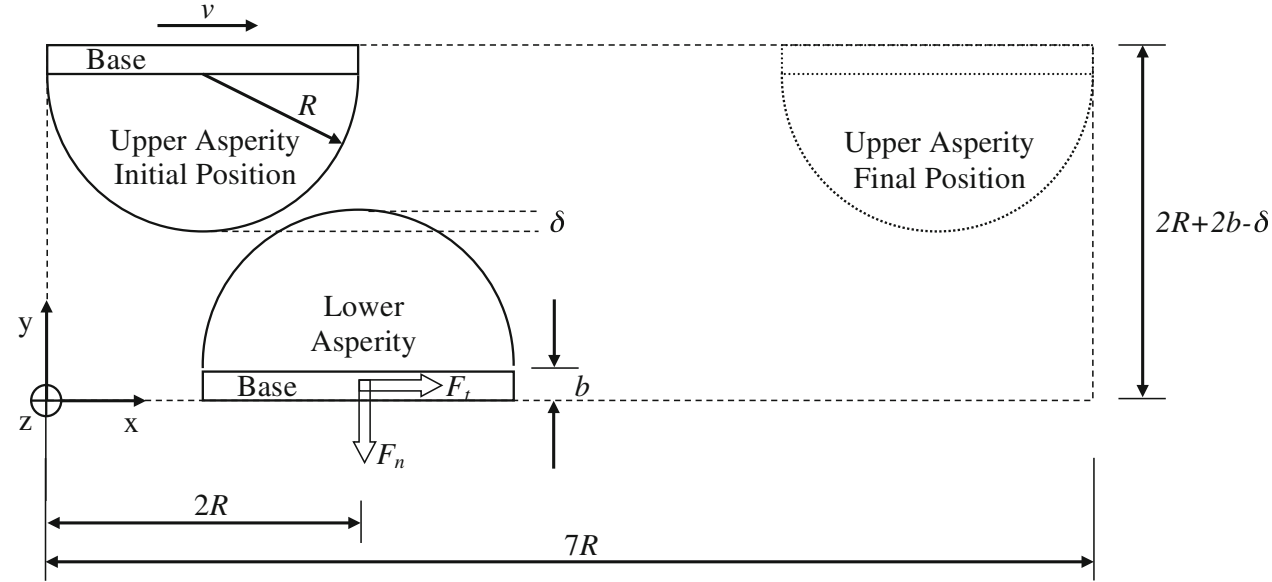

Table 1 Computation parameters for simulations

\begin{tabular}{llll}
\hline & $R=5 \mathrm{~nm}$ & $R=7.5 \mathrm{~nm}$ & $R=10 \mathrm{~nm}$ \\
\hline$b$ & $0.5 \mathrm{~nm}$ & $0.5 \mathrm{~nm}$ & $0.5 \mathrm{~nm}$ \\
$\delta / R$ & 0.10 to -0.04 & 0.10 to -0.04 & 0.10 to -0.04 \\
Base atoms & $\sim 4,600$ & $\sim 10,500$ & $\sim 18,400$ \\
Asperity atoms & $\sim 22,400$ & $\sim 75,000$ & $\sim 177,000$ \\
Total atoms & $\sim 54,000$ & $\sim 172,000$ & $\sim 390,800$ \\
\hline
\end{tabular}

of the boundary and re-enter on the other side. Non-periodic and shrink-wrapped (the extents of the boundary are set so as to include the atoms in that dimension no matter how far they move (Plimpton 1995) boundary conditions are imposed along the $y$-axis. The size of the model and the number of atoms in the simulation are chosen to obtain a reasonable balance between nano-scale representation and the computational time and power required. Copper asperities of radii 5, 7.5 and $10 \mathrm{~nm}$ were used in this work and the number of corresponding atoms and system dimensions are given in Table 1.

The atomic interactions were described using the embedded atom method (EAM) given by Daw and Baskes (1984) provided in the LAMMPS library. The EAM treats each atom as embedded in a host lattice consisting of all other atoms where the total energy of a system with $N$ atoms is given by,

$E_{\text {tot }}=\sum_{i=1}^{N} G_{i}\left[\sum_{j \neq i}^{N} \rho_{j}\left(r_{i j}\right)\right]+\frac{1}{2} \sum_{i, j \neq i}^{N} U_{i j}\left(r_{i j}\right)$.

Here, $G_{i}$ is the energy required to embed atom $i$ into electron density $\rho_{j}$ created by surrounding atoms and $U_{i j}\left(r_{i j}\right)$ is the electrostatic pair-potential between atoms $i$ and $j$ separated by distance $r_{i j}$. In metals, since the valence electrons may be shared between atoms, local electron densities need to be taken into account and the bonds between atoms are not independent of each other.
Therefore, density-independent pair-potentials like the Lennard-Jones (LJ) potential are not always suitable to capture the physics of metallic bonding (Foiles et al. 1986). The EAM effectively includes the many-body contributions and allows the electron density to vary between surface and the bulk, and as a result accurately describes bulk and defect properties (energy, structural, mechanical, and thermal properties) of metals (specially, fcc metals; Foiles et al. 1986) and metal alloys (Plimpton and Hendrickson 1993, Plimpton 1995) when compared to other interatomic potentials. It should also be noted that EAM only accounts for the contribution of bound electrons to atomic potentials and not that of the valence electrons. An important difference of the current work compared to that of Zhong et al. (2003) is that in latter's work a generic $\mathrm{LJ}$ potential was used for the hard (non-deformable) upper asperity and the EAM potential was used for the lower $\mathrm{Al}$ asperity.

Newton's equations of motion were numerically integrated using the velocity-Verlet algorithm with a time-step size of $0.002 \mathrm{ps}$. The time-step was empirically determined to capture the system dynamics as best as possible while keeping the computational time reasonable. The MD simulations were performed in two stages on a high-performance computing cluster using between 40 and 80 , $2.8 \mathrm{GHz}$ Intel Xeon processors in parallel. First, for each simulation run, the system was initialized and equilibrated at $300 \mathrm{~K}$ temperature for $10 \mathrm{ps}(5,000$ time-steps). For the remaining time of the simulation, the temperature control was not enforced anywhere on the system. However, it was found that holding the temperature constant on the base only affected the effective friction coefficient in a minor way (as shown in a later section). After the equilibrating cycle, the top asperity was set in motion towards the bottom asperity by imposing an average velocity on the group of atoms in the base region along the $x$-direction. During the asperity interaction, normal and tangential reaction 
forces were monitored on the base regions. These forces were averaged over the sliding distance and an effective friction coefficient was calculated for the distance through which the asperities were in contact, given by

$$
F=\frac{1}{N} \sum_{i=n_{i}}^{n_{o}} f_{i}
$$

$\mu_{\mathrm{eff}}=\frac{F_{t}}{F_{n}}$.

Here, $n_{i}$ and $n_{o}$ are the force values corresponding to the time when the asperities come in contact and get out of contact, respectively and $N$ is the number of force values between $n_{i}$ and $n_{o}$. Note that $\mu_{\text {eff }}$ is not the same as the friction coefficient measured at the macro scale. However, they may correlate if an average is taken for different asperity sizes at different interferences.

\section{Results and discussion}

\section{Asperity-asperity interaction}

In order to obtain the sliding motion of the asperities across each other, the atoms in the base region of the upper asperity were imposed with a velocity in the $x$-direction ([100] direction). The lower asperity was held fixed to its position while the position of the upper asperity was set to get interference with the lower asperity for a range of $0.1 R$ to $-0.04 R$. The $\delta / R$ values of less than zero were included for two reasons: (1) at theoretical zero interference, a positive interference was generated when the system was equilibrated at $300 \mathrm{~K}$ as the atoms relaxed and (2) the adhesion was strong enough to pull the atoms to make contact if the two asperity tips were within the attractive range. The simulations were run for a sufficient number of time-steps to ensure that the asperities were completely out of contact. At every time-step, the forces on the atoms in the base regions were summed and recorded to obtain the reaction force components. These values of forces were averaged for the time the asperities remained in contact. A visualization tool was used to note the start and end of the contact and this was also verified by the values of forces, which averaged to zero before and after the contact.

Figure 2 shows the asperities at the start and end of sliding contact for $\delta / R=0.10$ and $v=10 \mathrm{~m} / \mathrm{s}$ for the asperity of radius $5 \mathrm{~nm}$. In the first part of the sliding process, the upper asperity makes contact with the lower one and a junction is formed. This junction grows through the sliding as more atoms bond to each other and the free surface decreases until the centers of the asperities align. In the second part, this adhered junction starts stretching along the sliding direction as the asperities move apart and finally breaks after necking. Such formation of necking is consistent with the work by Zhong et al. (2003). They report that the neck glides on the Al surface along a favorable slip system and material is transferred from lower $\mathrm{Al}$ tip to the upper $\mathrm{LJ}$ tip by adhesion. It can be seen (in Fig. 2) that in the current work both the asperities undergo severe plastic deformation along with several atoms being transferred from one asperity to the other.

Figure 3 shows the friction force which starts from zero, reaches a maximum at approximately $x / R=0.75$ soon after the asperities align and decreases back to zero as the asperities come out of contact. The first half of the sliding process is characterized mostly by the ploughing of atoms while the second half is dominated by adhesion. This is further supported by the fact that the normal force, as shown in Fig. 4, starts increasing as the asperities come into contact and peaks approximately when the upper and the lower asperities align at $x / R=0.0$. It then starts decreasing and eventually changes direction. Even before making a contact, as the asperities get closer the adhesion force pulls them into contact. This is also seen at $x / R$ values between -0.75 and -0.50 . At the micro-scale, the inverse trend has been observed for the friction and the normal forces (Jackson et al. 2007; Faulkner and Arnell 2000). In the elasto-plastic sliding of micro scale hemispherical asperities without adhesion, it is the friction force that changes direction as the asperities push each other apart at the end of the contact. The normal force does not show this behavior due to the absence of a strong adhesion force. However, here the adhesion was strong enough to pull the atoms of the asperities to make contact even when the interference was negative, i.e. the asperities were separated by a distance of $0.04 R$. Similar behavior was observed for copper asperity sliding on a copper work piece where strong adhesion resulted in larger contact area (Zhang et al. 2001; Cha et al. 2004; Li et al. 2003).

\section{Effect of interference, $\delta$}

Figure 5 shows the effect of normalized interference $(\delta / R)$ on the averaged friction force for all three asperity sizes and relative sliding velocities. Here, the interference $\delta$, is normalized by the asperity radius $R$ and the interference values used in the current work is in the range $0.1 \delta / R$ to $0.04 \delta / R$. This range of interferences is similar to that encountered for the single asperities in rough surface contacts. For example, large deformations are usually not as statistically significant in a rough surface contact due to there being very few of them (Jackson and Green 2006).

As the interference increases, the number of atoms, which interact with each other, also increases. This causes increased atom displacement as well as adhesion and results in an increased friction force, which is needed to 
Fig. 2 Copper asperities before (left) and after (right) sliding process for $R=7.5 \mathrm{~nm}$, $\delta / R=0.10$ and $v=10 \mathrm{~m} / \mathrm{s}$
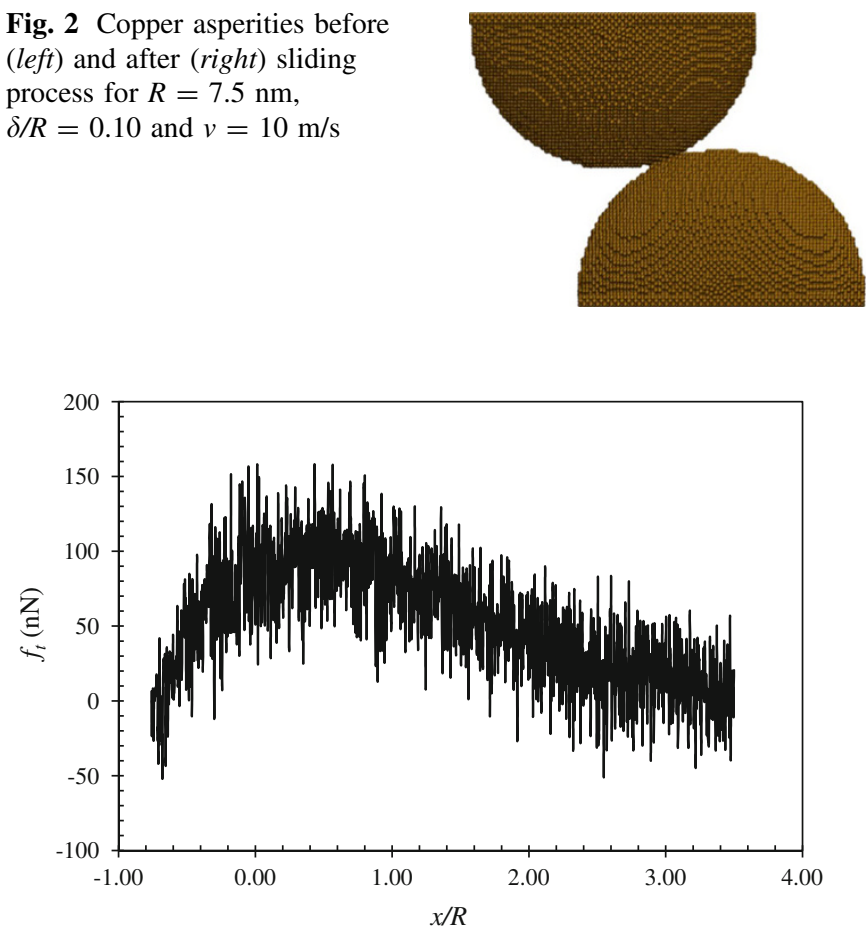

Fig. 3 The friction force during sliding in normalized sliding direction for $R=7.5 \mathrm{~nm}, \delta / R=0.10$ and $v=10 \mathrm{~m} / \mathrm{s}$

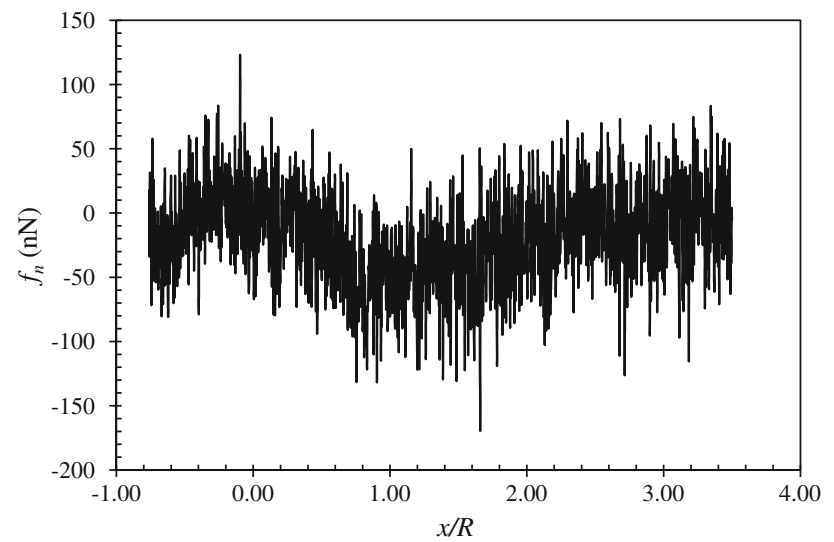

Fig. 4 The normal force during sliding in normalized sliding direction for $R=7.5 \mathrm{~nm}, \delta / R=0.10$ and $v=10 \mathrm{~m} / \mathrm{s}$

plough through the atoms and also overcome the adhesion. Thus, the friction force is directly proportional to the interference. This is not the case with the normal force (see Fig. 6). The normal force, while not showing a definite trend, seems to randomly fluctuate for certain interference values, which are different for each asperity size. This also results in a fluctuation of the effective coefficient of friction with interference $(\delta / R)$. The visualizations of the sliding process were analyzed which revealed that this fluctuation was caused due to the vertical alignment of the lattice and the resulting discretization of the smooth surface geometry

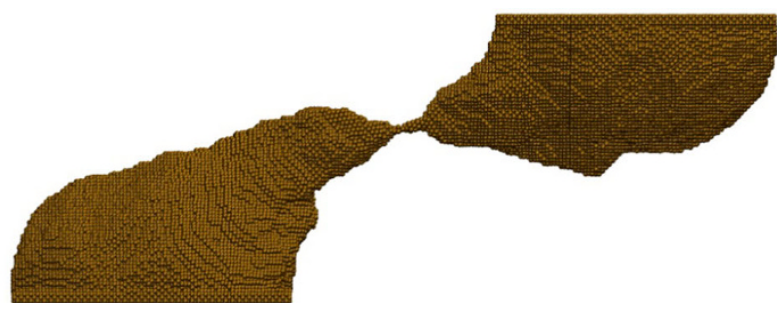

for a given value of interference. However, the asperities have the same average continuous geometry for all the values of interference. The number of atoms would either increase or decrease slightly depending on the discretization and result in a biased value of the normal force.

Similar to the current work, the results in a study of friction in nanometric scratching of a rigid diamond tool on a copper work-piece (Zhu et al. 2011) show a steeper increase in the friction force as compared to the normal force with increasing scratching depth. That work reports that at smaller scratching depths friction was dominated by adhesion. In this work since the friction force is rapidly increasing compared to the normal force, the effective friction coefficient also increases with the interference (see Fig. 7).

Effect of asperity radius, $R$

To study the effect of asperity size on the forces and effective friction coefficient, asperities with radii of 5, 7.5, and $10 \mathrm{~nm}$ were considered. As mentioned earlier, these asperity sizes are chosen to obtain a reasonable balance between nano-scale representation and the computational time and power required. The typical surface roughness of MEMS/NEMS surfaces (Bora et al. 2005; Rezvanian et al. 2007; Ansari and Ashurst 2011) was used as a reference for choosing these sizes.

Again, as the asperity size increased, the number of atoms in both of the asperities taking part in the interaction also increased. Note that as the size increases the smoothness of the curvature of the asperity also increases. This further adds to the number of interacting atoms. Figure 8 shows that the friction force and the normal force increase almost linearly with size. The effective coefficient of friction (Fig. 9) also increases with size since the friction force increases more rapidly than the normal force. This was expected and was in agreement to other similar works (Zhang et al. 2001; Stone et al. 2008).

Effect of sliding velocity, $v$

Three sliding velocities of 10,50 , and $100 \mathrm{~m} / \mathrm{s}$ were used in this work to quantify its effect on the friction characteristics along with the change in the interference and size. 
Fig. 5 The effect of interference, asperity size, and relative sliding velocity on the average friction force
Fig. 6 The effect of interference, asperity size, and relative sliding velocity on the average normal force
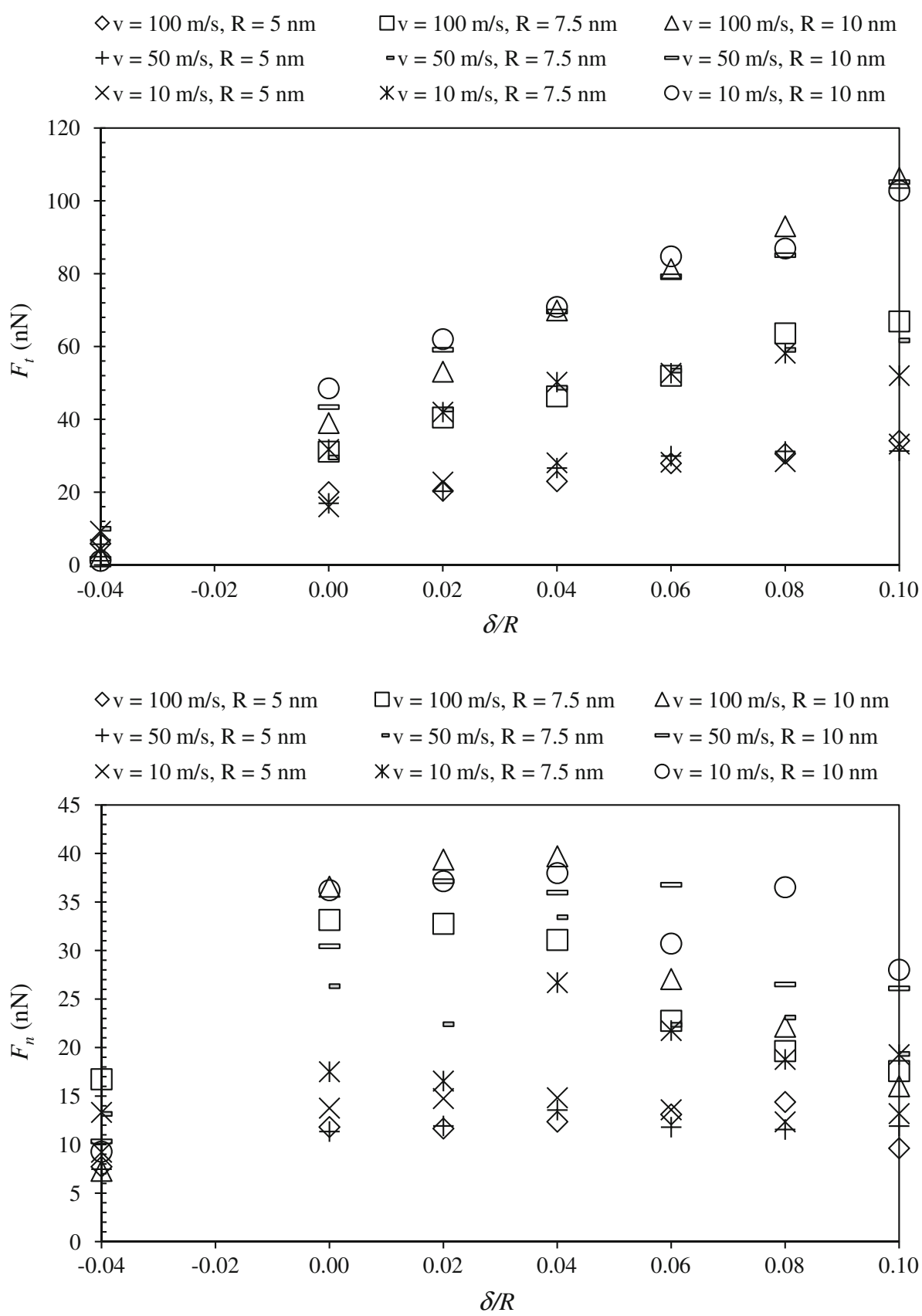

These values of velocity represent the velocities commonly encountered in MEMS/NEMS devices (Ping and NingBo 2007; Karthikeyan et al. 2009; Pei et al. 2007). For example, a high temperature micro gas turbine has a rotational speed that would translate into a sliding velocity of over $500 \mathrm{~m} / \mathrm{s}$ (Bhushan 2007).

An important observation of this work was that for the sliding velocity of 50 and $100 \mathrm{~m} / \mathrm{s}$, due to the sudden acceleration of the asperity atoms in the $\mathrm{x}$-direction at the start of the simulation, the upper asperity oscillated about its center as it travelled towards and across the lower asperity. This caused the normal force and as a result the friction coefficient to fluctuate severely. To reduce this effect, instead of applying an instantaneous increase in velocity, the atoms were gradually accelerated. A velocity ramping function $v(t)=\tan h(t / C)$, where $t$ is the time, and $C$ is the constant, was used to accelerate the asperity to 50 and $100 \mathrm{~m} / \mathrm{s}$ before the start of the contact and then was maintained at this velocity for the remaining sliding process. Although not evident during the visualization and in the force curves, some of this oscillation might still be present even after the gradual ramping of the velocity as well as for a sliding velocity of $10 \mathrm{~m} / \mathrm{s}$ for which such a ramping function was not in place. This could also explain 
Fig. 7 The effect of interference, asperity size, and relative sliding velocity on the effective friction coefficient

Fig. 8 The effect of asperity size on the average friction force and the average normal force, averaged over $\delta / R$
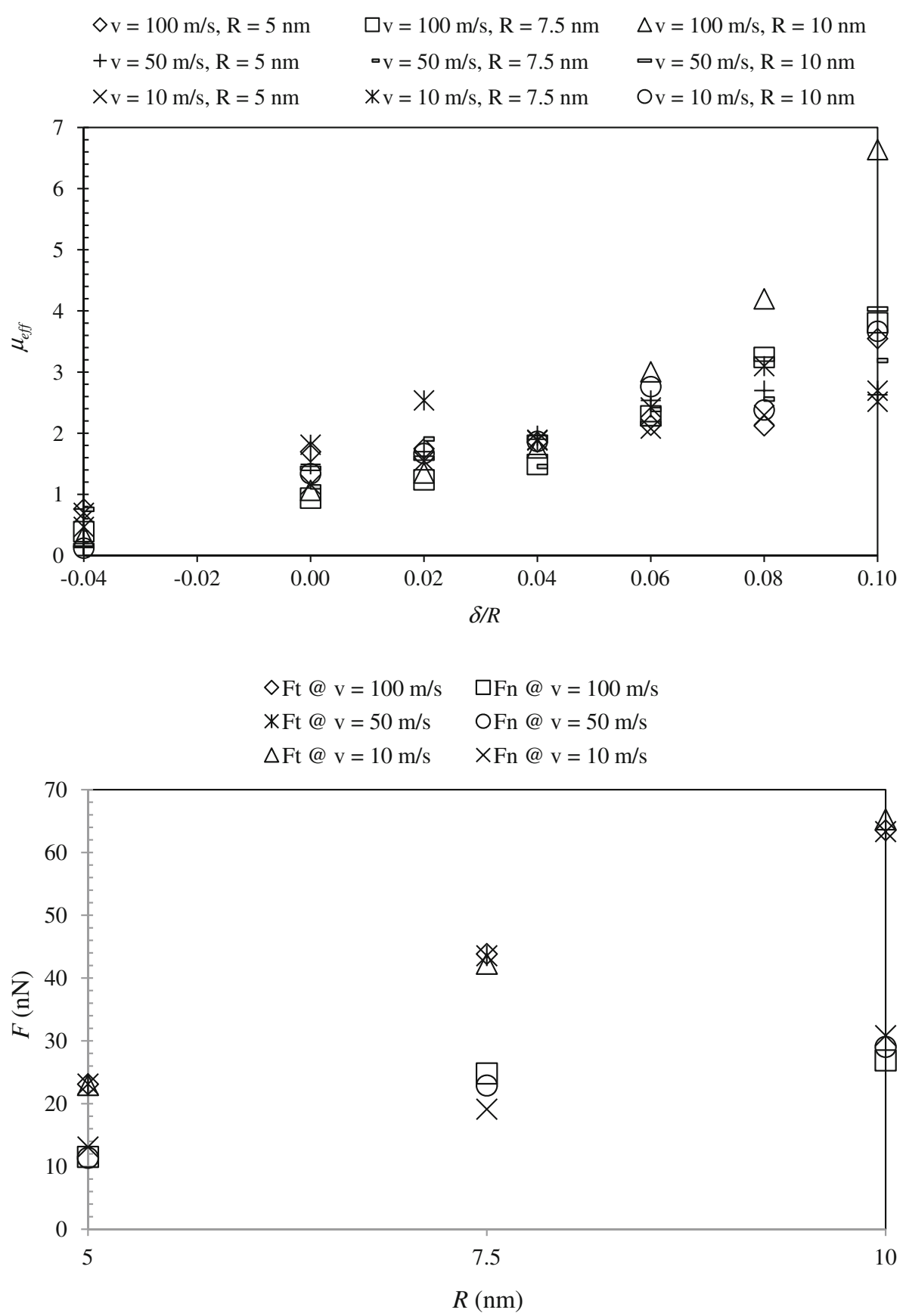

the random fluctuation of the normal force as observed in Fig. 6, but then one would also expect the tangential force to fluctuate and it does not. Therefore, it appears more likely that the previously mentioned lattice alignment effect causes these fluctuations.

As seen in Figs. 5, 6, 7, 8, 9, the change in the sliding velocity of the asperity did not produce any consistent and significant change in the forces and the effective friction coefficient. This is in contradiction to several reported results on asperity sliding friction (Yang and Komvopoulos 2005; Karthikeyan et al. 2009; Lin et al. 2007).
Karthikeyan et al. (2009) have reported a fivefold increase in the friction coefficient when the sliding velocity of copper block on an iron block was increased from 300 to $1,000 \mathrm{~m} / \mathrm{s}$. It should be noted that this sliding velocity is in a different range than that considered in the current work. In the sliding of a square prismatic diamond tip on a copper surface, the friction force increased as the sliding velocity increased from 10 to $100 \mathrm{~m} / \mathrm{s}$ while the normal force remained unchanged (Yang and Komvopoulos 2005). However, the main difference between the above-mentioned works and the current work is that the sliding 
Fig. 9 The effect of asperity size on the effective friction coefficient averaged over $\delta / R$

$$
\diamond_{\mathrm{v}}=100 \mathrm{~m} / \mathrm{s} \quad \Delta \mathrm{v}=50 \mathrm{~m} / \mathrm{s} \quad \square \mathrm{v}=10 \mathrm{~m} / \mathrm{s}
$$

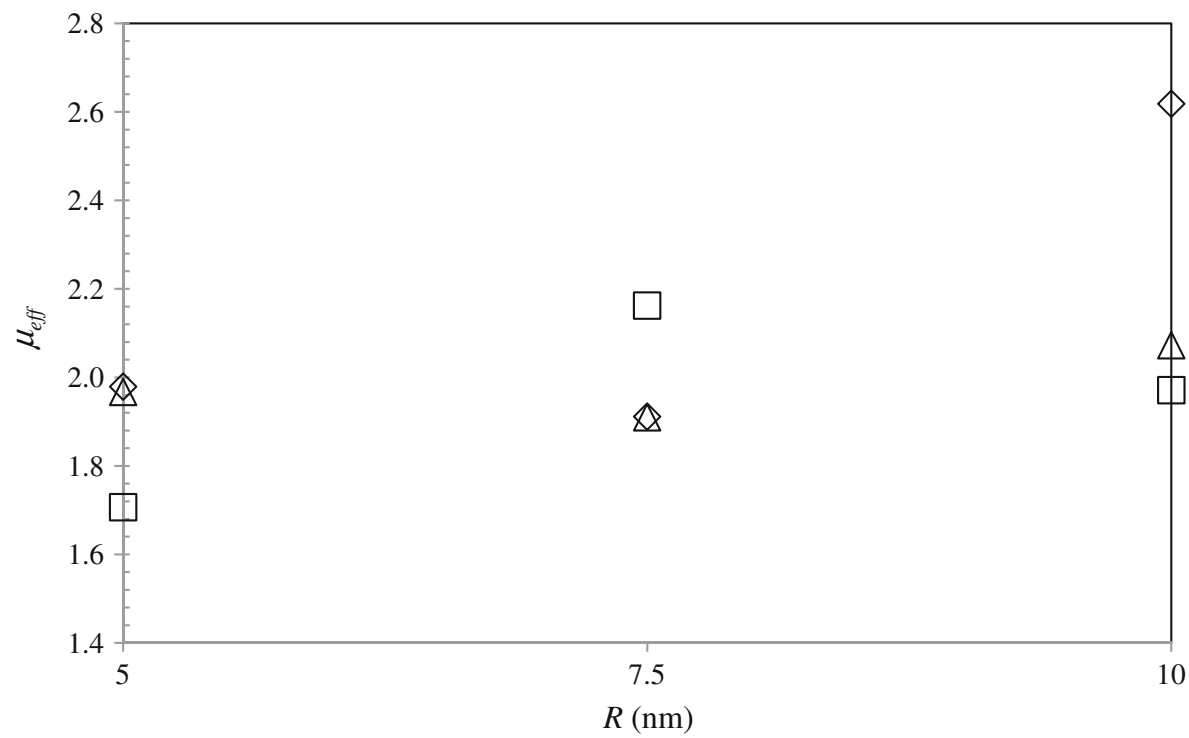

surfaces remain in contact for the entire duration and the contact area is also essentially constant throughout the sliding process in those works. In the current work, asperities remain in contact for only a small duration with the contact area continuously changing. Intuitively, this seems logical since there would be less time for the temperature to rise. In better agreement with the current work, Zhong et al. (2003) performed the simulations with sliding velocities of 50,100 , and $400 \mathrm{~m} / \mathrm{s}$ and found that at $300 \mathrm{~K}$ the wear of the aluminum asperity changed very little with respect to the sliding velocity.

Effect of lattice orientation, $\theta$

All of the simulations so far were performed with the $x$-, $y$ and $z$-axis oriented in the [100], [010] and [001] lattice directions, respectively, such that the (001) planes were parallel to the sliding direction. The normal force fluctuation perhaps caused due the vertical alignment of the lattices and the resulting discretization of the asperity surfaces for certain interference values was observed. If the orientation of the lattice is changed then this phenomena should disappear. This is demonstrated by performing simulations for various lattice orientations, $\theta$, about the $z$-axis as listed in Table 2. This is probably a more realistic condition since, in a real surface, the lattices are oriented randomly within the asperities and thus, would average out the orientation bias. The slip planes of both the asperities remained parallel to each other as the lattices were reoriented the same for both asperities.

As $\theta$ increased, the sliding direction appeared more favorable for slip and the friction force decreased while the normal force increased. The deformation or the material
Table 2 Lattice orientations simulated

\begin{tabular}{lc}
\hline$x y z$ direction & $\theta$ \\
\hline$[110],[\overline{1} 10],[001]$ & $45^{\circ}$ \\
{$[320],[\overline{1} 30],[001]$} & $34^{\circ}$ \\
{$[210],[\overline{1} 20],[001]$} & $27^{\circ}$ \\
{$[310],[\overline{1} 30],[001]$} & $18^{\circ}$ \\
{$[410],[\overline{1} 40],[001]$} & $14^{\circ}$ \\
\hline
\end{tabular}

transfer did not decrease visibly for the range $\theta=0^{\circ}$ to $\theta=34^{\circ}$. But at $\theta=45^{\circ}$, which corresponds to the (101) planes parallel to the sliding direction, the deformation decreased dramatically with much less material transfer as observed in Fig. 10. Zhong et al. (2003) also reported that the wear decreased significantly when the sliding surfaces where parallel to the (111) planes compared to the (100) planes. Further, Sorensen et al. (1996) have shown that between a $\mathrm{Cu}$ tip and $\mathrm{Cu}$ surface, with non-matching surfaces parallel to the (111) plane the wear is minimum. Although, the most favorable case of (111) parallel to the sliding direction is not considered in this work, the results are comparable. However, one should also note that the contact plane actually changes in the current work due to the curved nature of both surfaces (i.e. at the initial contact the plane between the two surfaces will be sloped, but as the asperities progress further, the slope eventually becomes zero). In most of the other works that are of an asperity against a flat, the contact plane is always parallel to the sliding plane.

Similar to the case of $\theta=0^{\circ}$, Fig. 11 shows the friction force for the case of $\theta=45^{\circ}$ which starts from zero, reaches a maximum as the asperities align and decreases 

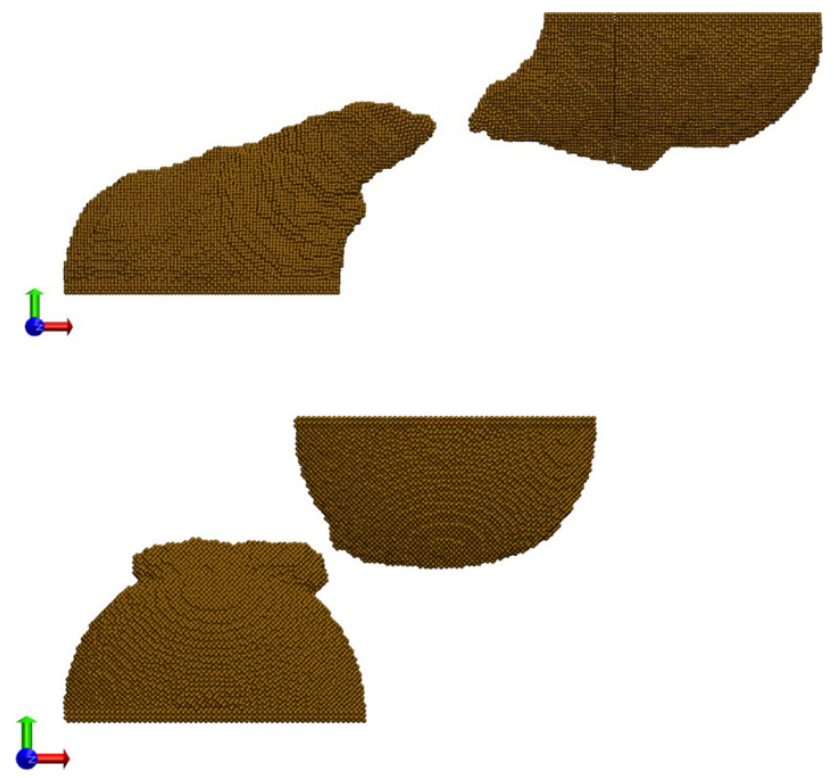

Fig. 10 Deformation at the end of sliding for $\theta=0^{\circ}$ (top), $\theta=45^{\circ}$ (bottom) for $R=7.5 \mathrm{~nm}, \delta / R=0.10$ and $v=10 \mathrm{~m} / \mathrm{s}$

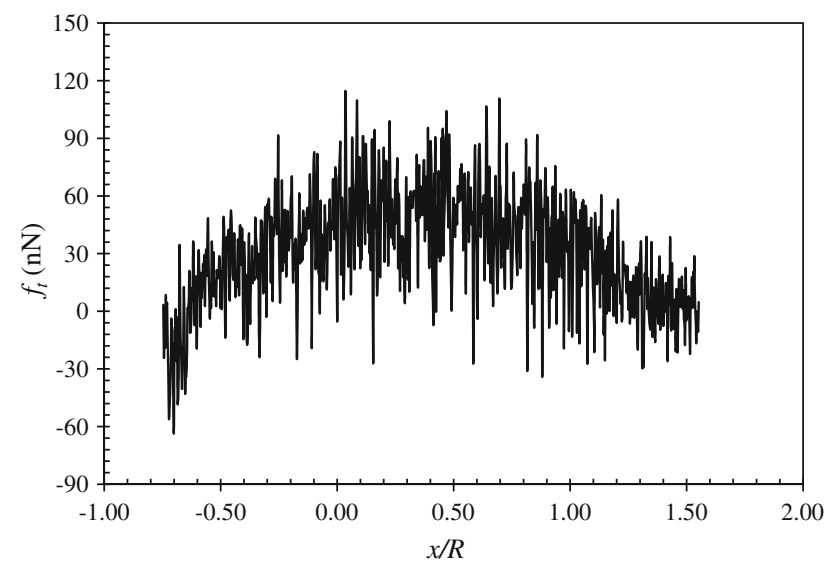

Fig. 11 The friction force during sliding in normalized sliding direction for $R=7.5 \mathrm{~nm}, \delta / R=0.10$ and $v=10 \mathrm{~m} / \mathrm{s}$ with $\theta=45^{\circ}$

back to zero as the asperities come out of contact. However, the normal force does not show the same nature in this case (see Fig. 12). First, the asperities do not get pulled into contact as they come closer and the corresponding jump in the normal force is also not observed. Second, the normal force increases to a maximum as the asperities align and decreases back to zero as the asperities come out of contact without changing the direction. This suggests that the adhesion is comparatively much lower when $\theta=45^{\circ}$ to significantly resist the separation of asperities than when $\theta=0^{\circ}$

Figure 13 shows the effect of lattice orientation on the effective friction coefficient for the asperity of radius $7.5 \mathrm{~nm}$ and a sliding velocity of $10 \mathrm{~m} / \mathrm{s}$. As the lattice is rotated about the $z$-axis from $0^{\circ}$ to $45^{\circ}$ the friction force

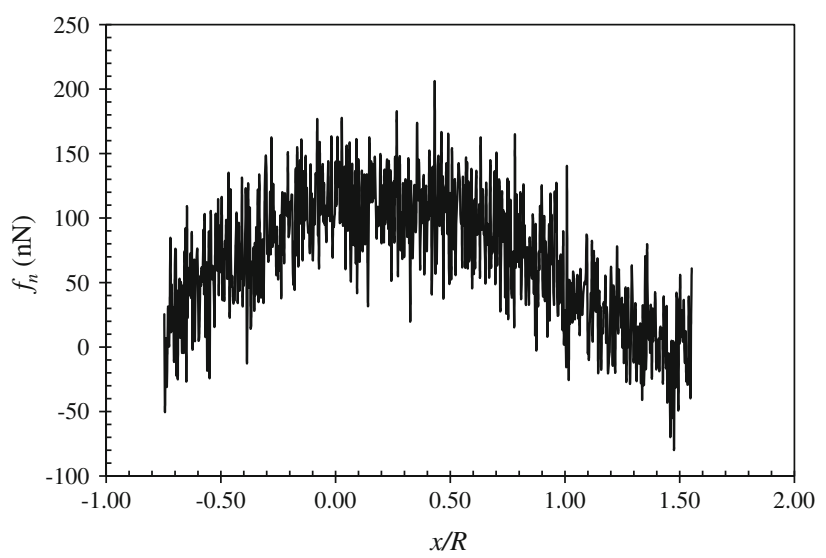

Fig. 12 The normal force during sliding in normalized sliding direction for $R=7.5 \mathrm{~nm}, \delta / R=0.10$ and $v=10 \mathrm{~m} / \mathrm{s}$ with $\theta=45^{\circ}$

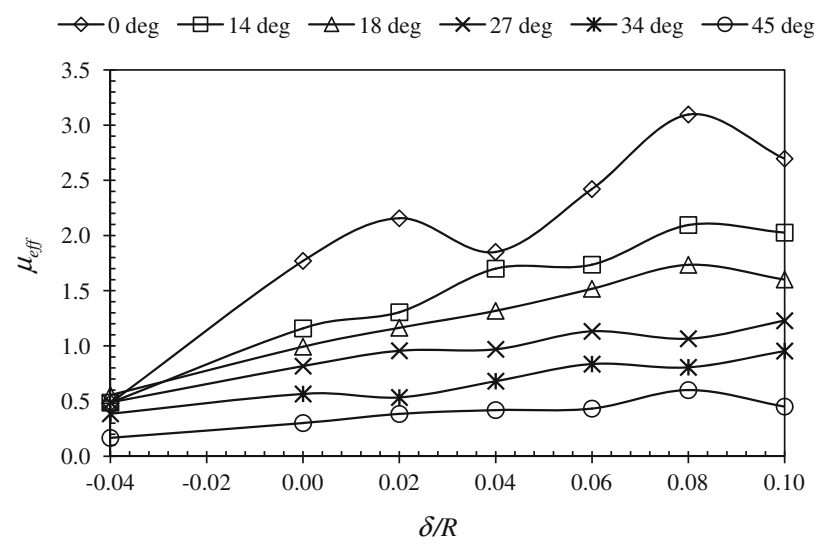

Fig. 13 The effect of lattice orientation on the effective friction coefficient for $R=7.5 \mathrm{~nm}, v=10 \mathrm{~m} / \mathrm{s}$

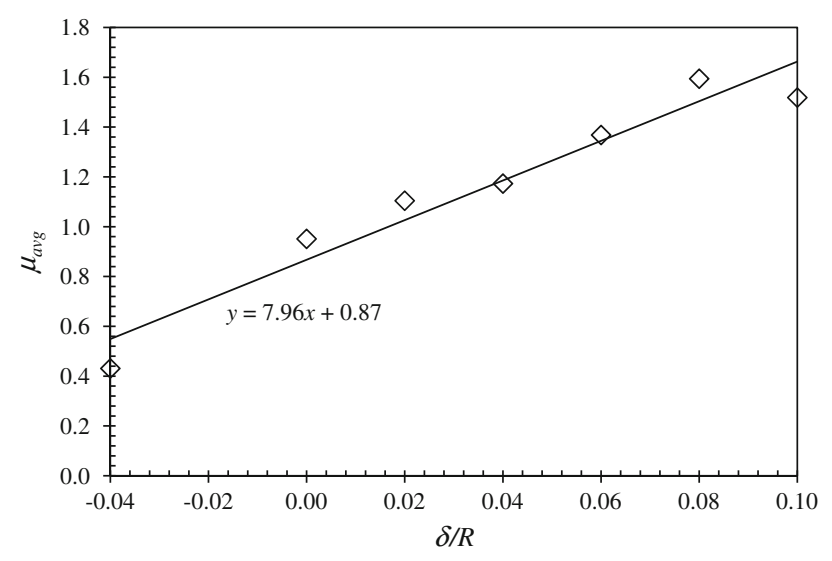

Fig. 14 The average effective friction coefficient as a function of normalized interference for $R=7.5 \mathrm{~nm}, v=10 \mathrm{~m} / \mathrm{s}$, solid line is a linear fit

decreases while the normal force increases and as a result the effective friction coefficient also decreases on average by a factor of about 6 . An average effective friction 
coefficient was calculated and plotted for the interference values considered as shown in Fig. 14. In some of the early experiments (Buckley 1967), the values of friction coefficient of pure polycrystalline copper in high vacuum were reported to be in the range 0.9-1.2. These are in reasonable agreement with values of $0.4-1.6$ obtained in the current work.

\section{Effect of temperature control}

All of the simulations performed up to this point in this work did not have any temperature control or thermostat in place during the sliding interaction, which would drain the energy out of the system. However, most of the works reported in the background section of this report had some sort of thermostat present in their system. Therefore, further simulations were carried out with a temperature control in order to observe its effect on the frictional characteristics. An asperity of radius $7.5 \mathrm{~nm}$ with a sliding velocity of $10 \mathrm{~m} / \mathrm{s}$ was simulated for two different lattice orientations: $\theta=0^{\circ}$ and $\theta=45^{\circ}$. After the entire system was equilibrated at $300 \mathrm{~K}$, the base regions of both the lower and the upper asperities were held at $300 \mathrm{~K}$ by scaling the velocities of the atoms.

Figure 15 shows the effective friction coefficient as a function of interference with and without a thermostat. It can be seen that there was little overall change observed by putting a temperature control for both the lattice orientations. Further, it confirms the previous finding of a lower effective friction coefficient at $\theta=45^{\circ}$. The effect of temperature control on the asperity temperature for lattice orientation of $\theta=0^{\circ}$ and $\theta=45^{\circ}$ is shown in Fig. 16. When the thermostat was not in place, the asperity temperature increased by approximately $80 \mathrm{~K}$ for the case of $\theta=45^{\circ}$ as compared to $230 \mathrm{~K}$ for $\theta=0^{\circ}$ since the work required to slide the asperities across each other is more in

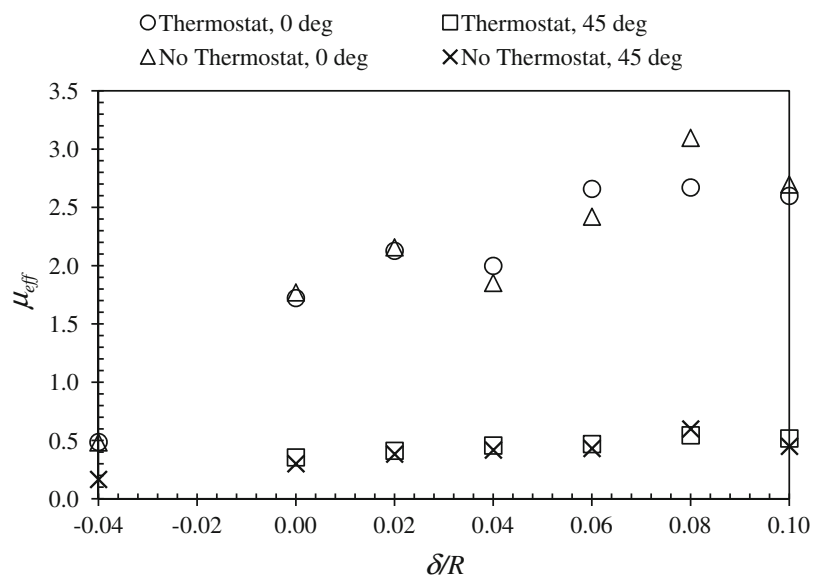

Fig. 15 The effect of temperature control on the effective friction coefficient for $R=7.5 \mathrm{~nm}, v=10 \mathrm{~m} / \mathrm{s}$

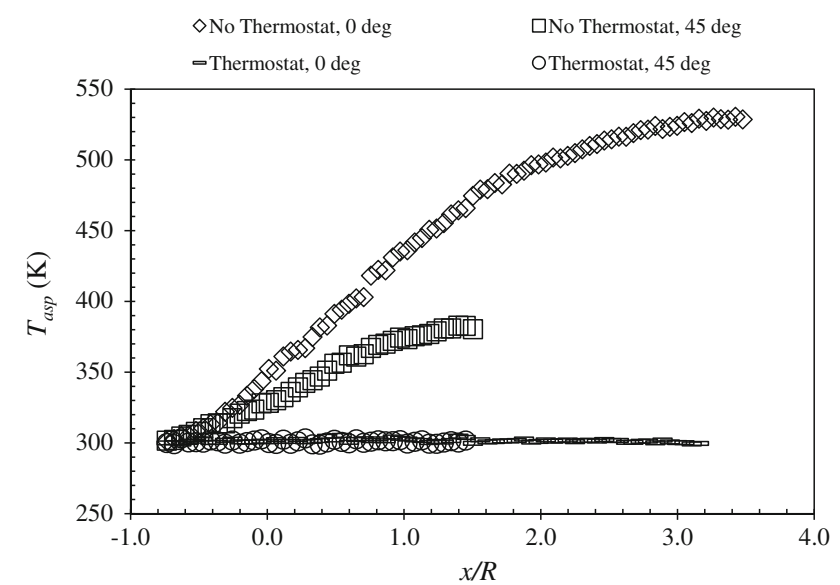

Fig. 16 The effect of temperature control and lattice orientation on the asperity temperature for $R=7.5 \mathrm{~nm}, v=10 \mathrm{~m} / \mathrm{s}$

the later case. Thus, the temperature rise is a function of adhesion and this finding is in agreement with the work by Ray and Roy Chowdhury (2010). Also, the duration for which the asperities remained in contact is also much smaller for $\theta=45^{\circ}$ for the same reason. This was also the case when the thermostat was in place, however, there was no significant increase in the asperity temperature for either of the orientations.

Electrons and phonons carry out thermal transport in metals, and for pure bulk metals the phononic contribution can be negligible. At the nano scale, the thermal transport decreases due to boundary scattering of electrons and phonons, and size effects (Yuan and Jiang 2006). Since the mean free path for electrons is larger than that of phonons, electrons suffer more scattering than phonons and therefore their contribution to thermal transport decreases (Feng et al. 2009). Even then the contribution of conducting electrons is crucial in thermal transport. As mentioned earlier, although EAM describes metallic interactions effectively it neglects the contribution of conducting electrons and therefore under-predicts the thermal transport. Consequently, the temperature rise observed as mentioned above is probably over predicted, and yet its effect is still relatively small on the friction coefficient.

\section{Conclusions}

Molecular dynamics simulations of the dry sliding of copper asperities were performed using the embedded atom potential with LAMMPS. The aim of this work was to study asperity-asperity interaction to gain useful insights on the deformation mechanisms and frictional characteristics in a dry sliding process with a geometry that could be compared and perhaps coupled to continuum models. The 
effect of interference, asperity size, sliding velocity, lattice orientation and temperature control on sliding friction was investigated. The important findings and conclusions are summarized as follows:

1. As the asperities come in contact a junction was formed which grew through the sliding as more atoms bonded to each other. This adhered junction stretched along the sliding direction as the asperities moved apart and finally broke after necking. Extensive deformation and material transfer was observed for most of the cases that were studied.

2. Adhesion dictated the sliding process as opposed to ploughing resulting in higher values of effective friction coefficient, which is consistent with literature.

3. Friction force and effective friction coefficient increased with the interference whereas the friction force, normal force, and effective friction coefficient increased with the asperity size. This is attributed to the increase in the number of interacting atoms. Fluctuations in the normal force were observed due to the vertical lattice alignment and the resulting discretization of the smooth surface geometry for a given value of interference.

4. For the range of $10-100 \mathrm{~m} / \mathrm{s}$, the velocity presented no significant change in the friction characteristics as the duration of contact was not long enough to realize its effect.

5. Lattice orientation presented a significant influence on the frictional characteristics with a reduction in the effective friction coefficient by a factor of about 6 for the range of orientation considered. For the case when the sliding direction was parallel to the (101) plane, the least material transfer and deformation were observed.

6. Using a temperature control during the sliding with the base of the asperities at $300 \mathrm{~K}$ produced almost no change in the friction characteristics in comparison to no temperature control.

Acknowledgments We would like to thank Steve Plimpton of Sandia National Laboratories and Robert S. Hoy of the Department of Mechanical Engineering and Material Science at Yale University for their valuable suggestions on some details of the simulations.

Open Access This article is distributed under the terms of the Creative Commons Attribution License which permits any use, distribution, and reproduction in any medium, provided the original author(s) and the source are credited.

\section{References}

Achanta S, Liskiewicz T, Drees D, Celis J (2009) Friction mechanisms at the micro-scale. Tribol Int 42:1792-1799
Ansari N, Ashurst WR (2011) An easy-to-fabricate improved microinstrument for systematically investigating adhesion between MEMS sidewalls. Appl Surf Sci 257:10917-10925

Barriga J, Fernandez-Diaz B, Juarros A, Ahmed SI-U, Arana JL (2007) Microtribological analysis of gold and copper contacts. Tribol Int 40:1526-1530

Bhushan B (2007) Nanotribology and nanomechanics of MEMS/ NEMS and BioMEMS/BioNEMS materials and devices. Microelectron Eng 84:387-412

Blau PJ (1991) Scale effects in steady-state friction. Tribol Trans 34(3):335-342

Bora CK, Flater EE, Street MD, Redmond JM, Starr MJ, Carpick RW et al (2005) Multiscale roughness and modeling of MEMS interfaces. Tribol Lett 19(1)

Boucly V, Nelias D, Green I (2007) Modeling of the rolling and sliding contact between two asperities. J Tribol 129:235-245

Buckley DH; Lewis Research Center, Ohio (1967) Effect of recrystallization on friction properties of some metals in single-crystal and polycrystalline form. Technical Note, National Aeronautics and Space Administration

Cha P, Srolovitz DJ, Vanderlick TK (2004) Molecular dynamics simulation of single asperity contact. Acta Mater 52:3983-3996

Cho MH, Kim SJ, Lim D, Jang H (2005) Atomic scale stick-slip caused by dislocation nucleation and propagation during scratching of a $\mathrm{Cu}$ substrate with a nanoindenter: a molecular dynamics simulation. Wear 259:1392-1399

Choi Y, Lee C, Hwang Y, Park M, Lee J, Choi C et al (2009) Tribological behavior of copper nanoparticles as additives in oil. Curr Appl Phys 9:e124-e127

Daw MS, Baskes MI (1984) Embedded-atom method: derivation and application to impurities, surfaces and other defects in metals. Phys Rev B 29(12):6443-6453

Eid H, Adams GG, McGruer NE, Fortini A, Buldyrev S, Srolovitz D (2011) A combined molecular dynamics and finite element analysis of contact and adhesion of a rough sphere and a flat surface. Tribol Trans 54:920-928

Faulkner A, Arnell RD (2000) The development of a finite element model to simulate the sliding interaction between two, threedimensional, elastoplastic, hemispherical asperities. Wear 242:114-122

Feng B, Li Z, Zhang X (2009) Role of phonon in the thermal and electrical transport in metallic nanofilms. J Appl Phys 105:1-7

Foiles SM, Baskes MI, Daw MS (1986) Embedded-atom-method function for the fcc metals $\mathrm{Cu}, \mathrm{Ag}, \mathrm{Au}, \mathrm{Ni}, \mathrm{Pd}, \mathrm{Pt}$, and their alloys. Phys Rev B 33(12):7983-7991

Harrison JA, White CT, Colton RJ, Brenner DW (1992) Moleculardynamics simulations of atomic-scale friction of diamond surfaces. Phys Rev B 46(15):9700-9708

Ivashchenko VI, Turchi PE (2006) Atomic-scale sliding friction of amorphous and nanostructured $\mathrm{SiC}$ and diamond surfaces. Tribol Trans 49(1):61-65

Jackson RL (2006) The effect of scale-dependent hardness on elastoplastic asperity contact between rough surfaces. Tribol Trans 49(2):135-150

Jackson RL, Green I (2006) A statistical model of elasto-plastic asperity contact between rough surfaces. Tribol Int 39:906-914

Jackson RL, Duvvuru RS, Meghani H, Mahajan M (2007) An analysis of elasto-plastic sliding spherical asperity interaction. Wear 262:210-219

Jeng Y, Su C, Lay Y (2007) An investigation of nanoscale tribological characteristics under different interaction forces. Appl Surf Sci 253:6754-6761

Karthikeyan S, Agrawal A, Rigney DA (2009) Molecular dynamics simulations of sliding in an $\mathrm{Fe}-\mathrm{Cu}$ tribopair system. Wear 267:1166-1176 
Landman U, Luedtke WD, Gao J (1996) Atomic-scale issues in tribology: interfacial junctions and nano-elastohydrodynamics. Langmuir 12(19):4514-4528

Landman U, Gao J, Luedtke WD, Gourdon D, Ruths M, Israelachvili JN (2004) Frictional forces and Amontons' law: from the molecular to the macroscopic scale. J Phys Chem B 108(11):3410-3425

Li B, Clapp PC, Rifkin JA, Zhang XM (2003) Molecular dynamics calculation of heat dissipation during sliding friction. Int $\mathrm{J}$ Heat Mass Transf 46:37-43

Lin J, Fang T, Wu C, Houng K (2007) Contact and frictional behavior of rough surfaces using molecular dynamics combined with fractal theory. Comput Mater Sci 40:480-484

Liu G, Li X, Qin B, Xing D, Guo Y, Fan R (2004) Investigation of the mending effect and mechanism of copper nano-particles on a tribologically stressed surface. Tribol Lett 17(4):961-966

Osterle W, Prietzel C, Kloss H, Dmitriev AI (2010) On the role of copper in brake friction materials. Tribol Int 43:2317-2326

Pei QX, Lu C, Lee HP (2007) Large scale molecular dynamics study of nanometric machining of copper. Comput Mater Sci 41:177-185

Ping Y, NingBo L (2007) Surface sliding simulation in micro-gear train for adhesion problem and tribology design by using molecular dynamics model. Comput Mater Sci 38:678-684

Plimpton S (1995) Fast parallel algorithms for short range molecular dynamics. J Comput Phys 117:1-19

Plimpton SJ, Hendrickson BA (1993) Parallel molecular dynamics with the embedded atom method. Materials theory and modelling. MRS Proceedings 291, Pittsburgh, p 37

Ray S, Roy Chowdhury SK (2010) An analysis of surface temperature rise at the contact between sliding bodies with small-scale surface roughness. Tribol Trans 53:491-501
Rezvanian O, Zikry MA, Brown C, Krim J (2007) Surface roughness, asperity contact and gold RF MEMS switch behavior. J Micromech Microeng 17

Song J, Srolovitz DJ (2007) Atomistic simulation of multicycle asperity contact. Acta Mater 55:4759-4768

Sorensen MR, Jacobsen KW, Stoltze P (1996) Simulations of atomicscale sliding friction. Phys Rev B 53(4):2101-2113

Stone TW, Horstemeyer MF, Hammi Y, Gullett PM (2008) Contact and friction of single crystal nickel nanoparticles using molecular dynamics. Acta Mater 56:3577-3584

Tarasov S, Kolubaev A, Belyaev S, Lerner M, Tepper F (2002) Study of friction reduction by nanocopper additives to motor oil. Wear 252:63-69

Yang J, Komvopoulos K (2005) A molecular dynamics analysis of surface interference and tip shape and size effects on atomicscale friction. J Tribol 127:513-521

Yuan SP, Jiang PX (2006) Thermal conductivity of small nickel particles. Int J Thermophys 27(2):581-595

Zhang L, Tanaka H (1997) Towards a deeper understanding of wear and friction on the atomic scale-a molecular dynamics analysis. Wear 211:44-53

Zhang LC, Johnson KL, Cheong WC (2001) A molecular dynamics study of scale effects on the friction of single-asperity contacts. Tribol Lett 10(1-2):23-28

Zhong J, Adams JB, Hector LG (2003) Molecular dynamics simulations of asperity shear in aluminum. J Appl Phys 94(7):4306-4314

Zhu P, Hu Y, Ma T, Wang H (2011) Molecular dynamics study on friction due to ploughing and adhesion in nanometric scratching process. Tribol Lett 41:41-46 